\title{
Del aprendizaje en escenarios presenciales al aprendizaje virtual en tiempos de pandemia
}

\author{
From face-to-face learning to virtual learning in pandemic times
}

\section{Do aprendizado em sala de aula ao aprendizado virtual em tempos de pandemia}

\section{Floralba del Rocío Aguilar Gordón ${ }^{a}$}

${ }^{a}$ Universidad Politécnica Salesiana.

Grupo de Investigación Filosofía de la Educación (GIFE), Quito, Ecuador.

faguilar@ups.edu.ec

\section{RESUMEN}

El confinamiento obligó a la humanidad a efectuar transformaciones pedagógicas y sociales radicales, generó nuevos escenarios y modos de vida en los que prima el uso de nuevas tecnologías; los espacios físicos fueron reemplazados por espacios virtuales; la telemática se convirtió en el nuevo canal de comunicación e información, se forjaron el teletrabajo y la teleeducación. El objetivo de este documento es reflexionar sobre las implicaciones del paso del aprendizaje en escenarios presenciales al aprendizaje virtual en tiempos de pandemia; se propone conocer las distintas realidades de la comunidad educativa mediante la revisión de datos sobre deserción escolar, conectividad a internet, etc. Es una investigación de carácter descriptivo, puntualiza aspectos educativo-sociales ocurridos en los ambientes virtuales durante la emergencia sanitaria. La recolección de información se realizó mediante la búsqueda bibliográfica-documental alimentada desde la observación-experiencia directa del contexto social. Esta investigación se auxilió del método hermenéutico que propicia la interpretación de la información, de la realidad contextual y de las causas de la desigualdad social. El presente trabajo se encuentra dividido en cinco partes: la primera parte, describe la importancia de la educación en escenarios virtuales; la segunda parte, narra el aprendizaje en las aulas virtuales; la tercera parte, reflexiona acerca de las desventajas del aprendizaje virtual; la cuarta parte, expone la educación virtual como factor de desigualdad social, y, finalmente, presenta los retos del aprendizaje virtual en tiempos de pandemia.

Palabras claves: Aprendizaje, aprendizaje virtual, desigualdad social, pandemia, tecnología.

\begin{abstract}
Confinement forced humanity to carry out radical pedagogical and social transformations, generated new scenarios and ways of life in which the use of new technologies prevailed; physical spaces were replaced by virtual spaces; Telematics became the new channel of communication and information, telework and tele education were forged. The objective of this document is to reflect on the implications of the shift from face-toface learning to virtual learning in times of pandemic; aims to understand the different realities of the educational community by reviewing data on school dropout, internet connectivity, etc. It is a descriptive research, it points out educational-social aspects that occurred in virtual environments during the health emergency. The collection of information was carried out through the bibliographic-documentary search fed from the direct observationexperience of the social context. The present work is divided into five parts: the first part, describes the importance of education in virtual scenarios; the second part, narrates learning in virtual classrooms; the third part, reflects on the disadvantages of virtual learning; The fourth part exposes virtual education as a factor of social inequality, and finally presents the challenges of virtual learning in times of pandemic.
\end{abstract}

Key words: Learning, virtual learning, social inequality, pandemic, technology. 


\section{RESUMO}

O confinamento obrigou a humanidade a realizar transformações pedagógicas e sociais radicais, gerou novos cenários e modos de vida em que prevaleceu o uso de novas tecnologias; os espaços físicos foram substituídos por espaços virtuais; a telemática passou a ser o novo canal de comunicação e informação, o teletrabalho e a tele-educação foram forjados. O objetivo deste documento é refletir sobre as implicações da transição da aprendizagem em sala de aula para a aprendizagem virtual em tempos de pandemia; Propõe-se conhecer as diferentes realidades da comunidade educacional, analisando dados sobre evasão escolar, conectividade com a Internet, etc. É uma pesquisa descritiva, que especifica aspectos educacionais-sociais ocorridos em ambientes virtuais durante a emergência de saúde. A coleta de informações foi realizada por meio da pesquisa bibliográficadocumental alimentada a partir da observação-experiência direta do contexto social. Esta pesquisa foi auxiliada pelo método hermenêutico que privilegia a interpretação da informação, da realidade contextual e das causas da desigualdade social. Este trabalho está dividido em cinco partes: a primeira descreve a importância da educação em ambientes virtuais; a segunda parte narra a aprendizagem em salas de aula virtuais; a terceira parte reflete sobre as desvantagens da aprendizagem virtual; a quarta parte expõe a educação virtual como fator de desigualdade social e, por fim, apresenta os desafios da aprendizagem virtual em tempos de pandemia.

Palavras-chave: Aprendizagem, aprendizagem virtual, desigualdade social, pandemia, tecnologia.

\section{INTRODUCCIÓN}

El confinamiento social obligatorio causado por la pandemia modificó la forma de vida de los seres humanos, sin embargo, aunque el fenómeno viral llevó al aislamiento social, no significó una paralización absoluta de las actividades; al contrario, se incrementó el uso de medios tecnológicos que dio lugar al teletrabajo y la teleducación. La situación de la educación a nivel mundial durante la época de la pandemia se convirtió en un reto para los gobiernos y para la comunidad educativa, pues varias familias no contaban con los recursos necesarios para responder a las exigencias planteadas por el sistema educativo.

El aprendizaje virtual reemplazó drásticamente a los escenarios presenciales de aprendizaje. El sujeto educativo dejó de lado el contacto social para entrar en contacto directo con dispositivos digitales, la relación entre educador-educando se volvió asimétrica ya que la participación educativa no se dio en igualdad de condiciones. La realidad virtual introdujo nuevas formas de comprender el proceso educativo y produjo nuevos cuestionamientos como: ¿Qué tipo de aprendizaje genera la educación virtual? Y ¿Cuáles son las problemáticas sociales que surgen de este tipo de realidades virtuales?

\section{IMPORTANCIA DE LA EDUCACIÓN EN ESCENARIOS PRESENCIALES}

El proceso del aprendizaje ha ido evolucionando con el pasar de los siglos; sin embargo, es en la edad moderna en donde se formaliza la escolarización surgiendo interrogantes relacionadas con el proceso de enseñanza-aprendizaje: ¿Quién aprende? ¿Cuándo aprende? ¿Cómo se produce el aprendizaje? ¿Dónde se aprende? Tales preguntas son abordadas por varias disciplinas como la filosofía, la pedagogía, la psicología e incluso la sociología; de sus investigaciones o estudios nacen múltiples teorías, las cuales intentan definir de manera unívoca al aprendizaje.

Según Martí Castro (2003) el aprendizaje es comprendido como "el proceso mediante el cual se adquiere una determinada habilidad, se asimila una información o se adopta una nueva estrategia de conocimiento y acción" (p. 36), que genera transformaciones adaptativas en el sujeto y en el medio en el que se desarrolla. 
Es importante considerar que el proceso de aprendizaje va más allá de la educación escolar, pero es dentro del sistema educativo donde el sujeto aprende a interactuar con otros grupos que difieren en tradiciones, costumbres o creencias. La escuela se convierte en la institución capaz de formar integralmente al sujeto hasta que adquiera su condición ontológica de llegar a ser lo que es en relación con los otros.

El proceso de enseñanza-aprendizaje en los escenarios presenciales permite conocer las distintas realidades de los sujetos educativos, por ejemplo, la permanente convivencia del educador en el aula de clases le posibilita en términos de Narvarte (2008) reconocer "los trastornos que perturban el aprendizaje y detectar las posibles causas motoras, mentales, madurativas, emocionales, socioculturales" (p. 12) que afectan al educando; asimismo, a través de un equipo de orientación escolar se puede guiar a los padres de familia para una adecuada inclusión escolar.

Un aspecto positivo del aprendizaje en escenarios presenciales, según Papalia et al., (2012) es considerar que "el desarrollo humano es iniciado internamente por un organismo activo y ocurre en una serie de etapas diferenciales" (p. 25), el proceso de aprendizaje se adecúa a la edad del sujeto educativo y tal proceso es verificado mediante las actividades propuestas en el aula de clases y la evaluación de la ejecución de las mismas.

La importancia del proceso de aprendizaje en los escenarios presenciales radica según Albert Bandura (1925) en que "el motor de desarrollo tiene dos direcciones: la persona actúa en el mundo y el mundo actúa sobre ella" (citado en Papalia et al., 2012, p. 32), el comportamiento se da por los condicionamientos presentes en el ambiente, están ligados al reforzamiento. El sujeto no es un ser pasivo, es un ser capaz de transformar sus experiencias a través de la observación de modelos que influyen en sus motivaciones y en sus emociones. Por medio de este aprendizaje los estudiantes asimilan ciertas normas sociales que regulan su comportamiento.

Otro aspecto importante es la habilidad comunicacional entre el agente y el sujeto educativo, pues el proceso comunicacional favorece el trabajo cooperativo; en términos de Capdet (2011) "ya que a medida que se interactúa se va generando un clima de confianza y de seguridad que mejora el proceso comunicativo" (p. 43), las confusiones lingüísticas pueden ser manejadas entre el emisor y el receptor. El lenguaje en los espacios presenciales da lugar al contacto humano no solo por medio de palabras sino también por las expresiones corporales, expresadas a través de gestos, posturas o movimientos.

Además, es preciso considerar que la socialización, la diversidad social permite al educando desarrollar valores sociales y morales. En otras palabras, Tennuto et al., (2003) enfatizan que "en las instituciones educativas se generan espacios donde los actores sociales pueden establecer relaciones de cooperación, de solidaridad, de producción, de saberes y de acciones" (p. 42), mediante acuerdos o negociaciones que valoran distintos puntos de vista y que generan un espacio democrático capaz de manejar situaciones de conflicto.

\section{EL APRENDIZAJE EN LAS AULAS VIRTUALES}

El confinamiento obligó a la humanidad a adaptarse a un nuevo modo de vida que da lugar a la creación de nuevos escenarios en los que prima el uso de nuevas tecnologías que van transformando los espacios físicos, mismos que paulatinamente fueron reemplazados por espacios virtuales. La telemática se convirtió en un nuevo canal de comunicación e 
información que dio lugar al teletrabajo y a la teleeducación. El empleo de la telemática en el ámbito educativo, según Marti Castro (2003) es "cada vez más amplio y diverso, ya que la puede utilizar el profesor como soporte pedagógico o el alumno para el aprendizaje autónomo" (p. 406); sin embargo, si en las instituciones presenciales se evidenciaban fenómenos educativos relacionados con el acompañamiento escolar o educandos con problemas de aprendizaje, en el nuevo contexto, surge el siguiente cuestionamiento ¿Qué sucede con el aprendizaje en las aulas virtuales?

La inserción de las nuevas tecnologías de la información y de la comunicación (TIC) en el ámbito educativo, impactó en el proceso de aprendizaje del educando, en el rol del maestro, en los contenidos, en la evaluación. El papel del sujeto educativo afronta un periodo de crisis, pues por un lado genera aprendizajes significativos; y, por el otro lado, la falta de formación en las disciplinas da lugar a amplias brechas generacionales, que a decir de Aguilar-Gordón y Chamba (2019) "el analfabeto digital (docente) debe enseñar al erudito tecnológico del siglo XXI (estudiante)” (p. 115). Aun así, la innovación tecnológica en el sistema educativo se ha ido dando de manera gradual y de acuerdo a los recursos que cada institución educativa posee; no obstante, la pandemia actual provocada por la COVID19 obligó de manera abrupta a cambiar el proceso de enseñanza y aprendizaje presencial a un escenario virtual sin considerar el contexto de las comunidades educativas.

El aprendizaje virtual supone un cambio en la consolidación del conocimiento y la información a través de sistemas inteligentes, los cuales, conforme a Capdet (2011) sirven para "describir el contenido de los materiales educativos utilizados, monitorizar las entradas, salidas y actividades de los estudiantes, controlar que los trabajos se entregan en fecha, realizar la corrección automática de pruebas" (p. 50). El sujeto educativo pasa de ser consumidor a productor de información; el rol explicativo del docente ahora es comprendido como guía. El aprendizaje resulta significativo y a la vez, potencia el trabajo autónomo en el estudiante.

La educación virtual trae consigo beneficios a la comunidad educativa, permite desarrollar habilidades como la organización de información, el manejo de nuevos conceptos, la ampliación de lenguaje que favorece la comunicación y la conectividad. De acuerdo con Tennuto (2003) a través de la educación virtual "se pueden intercambiar varias unidades de información (gráficos, imágenes, archivos de sonido, bases de datos, entre otros" (p. 962) las cuales pueden ser obtenidas más allá de los espacios educativos. En este aspecto, el Internet proporciona infinitas posibilidades de conexión, da lugar al aprendizaje no formal e informal que parte de metodologías innovadoras ajustadas a las necesidades del educando. El aprendizaje virtual permite, en estos casos, establecer una mejor conexión con otros usuarios mediante diversos recursos: Blogs, Wikis, Twitter, Facebook, WhatsApp, y otros relacionados.

\section{DESVENTAJAS DE LA EDUCACIÓN VIRTUAL}

En la mayoría de los casos, la educación virtual en tiempos de pandemia no permite el acceso a un aprendizaje significativo ni mucho menos autónomo. La realidad que viven varias familias de clase media baja, sobre todo en América Latina deja ver la precariedad de las políticas educativas para la era digital. El repentino confinamiento condujo al ser humano a vivir de manera aislada y alterada, una realidad virtual cargada de lenguajes 
simbólicos propios de la inserción de la tecnología en los distintos aspectos del accionar humano y que poco a poco rompe con la concepción espacio-tiempo.

En la educación virtual cuando el sujeto se encuentra frente a otro solo puede percibir su mitad visible y en palabras de Zizek et al., (2008) "automáticamente lo des-subjetivamos llenando el vacío, proyectando en la oscuridad una riqueza de la personalidad imaginaria" (p. 20). La parte invisible del otro se convierte en un agujero vacío; por ello es necesario la re-significación del proceso educativo en escenarios virtuales; caso contrario solo se viviría de espejismos, presentando una incompletud ontológica de los sujetos.

La irrupción violenta de los escenarios virtuales, sobre todo en aquellos países que no se encontraban preparados para afrontar este tipo de emergencias, trae consigo una serie de dificultades políticas, económicas, psicosociales, educativas y culturales. La incorporación de dispositivos digitales (computadoras, laptops, celulares, smartphones, tablets) en el proceso de enseñanza y aprendizaje implica la creación de un nuevo entorno educativo, según expresa Tennuto (2003) se crea "un nuevo espacio formativo y de nuevas estructuras organizativas que requieren, por parte de los profesores y de los alumnos, actitudes favorables para interaccionar con este recurso tecnológico" (p. 962). Se reemplazó el aula de clases por espacios emergentes: dormitorio, comedor, sala, cuarto de estudio u otros similares; el contacto social entre compañeros de clases, amigos o docentes se limitó únicamente al contacto familiar. En este contexto surge una nueva interrogante: ¿Cómo la ausencia de contacto social incide en el aprendizaje?

A nivel mundial, el confinamiento obligatorio muestra la importancia de la escuela como espacio físico y el valor del docente que acompaña; sin esta presencia, varios niños y jóvenes son vulnerables frente a fenómenos como el suicidio, depresión, violaciones, violencia simbólica, violencia doméstica y deserción escolar. Los ambientes inseguros afectan el desarrollo emocional de la persona. Aristóteles (hacia 385 a.C.-323 a.C.) afirmaba que el ser humano es un ser social por naturaleza, él mismo construye su identidad desde los otros de manera subjetiva y cultural y si bien es cierto, la familia cumple un rol importante en la formación psicosocial del sujeto, éste siempre necesita de Otro externo como la escuela para confrontar y equilibrar sus creencias y costumbres.

Así mismo, en términos de Narvarte (2008) "en la escuela se puede trabajar el reconocimiento de las expresiones de sentimientos, ya sea con mímica o con dibujos" (p. 215). El aprendizaje en escenarios presenciales crea las condiciones necesarias para una sana convivencia. En el entorno virtual no se logra precisar este tipo de socialización, se dificulta el reconocimiento de algún tipo de trastorno psico-social o intelectual. Las aplicaciones virtuales presentan una realidad ficticia que impiden en su mayoría el manejo de las emociones, sea por la poca formación del docente en los recursos tecnológicos, la falta de recursos (dispositivos digitales, internet) por parte de la comunidad educativa, la falta de tiempo u otros factores. Al respecto, Zizek (2008) señala que "cuando vivimos en un espacio virtual aislado, toda reconexión con lo real es, (...) una experiencia demoledora; es violenta" (p. 74) porque el contacto social implica enfrentamientos y consensos. Las emociones deben ser trabajadas desde la primera infancia por el núcleo familiar, pero a veces la poca formación cultural de los padres impide desarrollarlas plenamente, la relación con otros pares diversos permite al educando aprender a ser afectivo, a expresar lo que siente, aceptarse a sí mismo mediante el asentimiento de los demás a través de etapas del conflicto.

El conócete a ti mismo proclamado por Sócrates (hacia 470 a.C.-379 a.C.) no es posible desde el aprendizaje virtual, la falta de contacto social impide que el sujeto logre 
una autoconciencia de sí mismo, debido a las distracciones provocadas por la cantidad de información o canales de entretenimiento que impiden el proceso reflexivo y la formación de un pensamiento crítico. Capdet (2011) sostiene que en la educación virtual "el usuario ha de aprender a interactuar con la máquina (software y hardware) y, una vez que lo ha conseguido, debe aprender a hacerlo con los usuarios conectados a la Red" (p. 12). Esta afirmación muestra como el sujeto educativo pierde su individualidad para diluirse en la generalización; el usuario es un navegante más en el ciber espacio, ya no existe un nombre o apellido propio que lo diferencie de los demás.

La educación virtual, desde Tennuto (2003) exige que el docente "desempeñe funciones que fundamentalmente girarán en torno a lo conceptual, a lo organizativo y a lo social" (p. 962), sólo así el sujeto que aprende puede responder con una mente crítica a las problemáticas sociales, desde la comunicación trasciende y puede concebirse como un ser ontológico que existe en un determinado tiempo-espacio como autor y actor del proceso educativo y de la misma historia. Además, como es de conocimiento general, el proceso de enseñanza y aprendizaje no es aislado, requiere de otras disciplinas que orienten al ser humano para la adquisición de nuevos referentes de comprensión desde una mirada antropológica y trascendental de la realidad; en este sentido, la filosofía de la tecnología, la antropología, la sociología, la psicología juegan un papel importante en la interacción de sujetos, ideas, contextos y circunstancias que trae consigo una riqueza de elementos culturales que humanizan los entornos virtuales y evitan la cosificación del ser humano.

\section{EDUCACIÓN VIRTUAL COMO FACTOR DE DESIGUALDAD SOCIAL}

La falta de contacto social en el aprendizaje virtual no es la única dificultad por la cual atraviesa la comunidad educativa, también es visible la violencia simbólica provocada por las desigualdades al momento de acceder a este tipo de aprendizaje. Antes que la pandemia irrumpiera la forma de vida de la humanidad, existían instituciones educativas que disponían de herramientas tecnológicas, existía personal capacitado en el uso se las TIC mientras que otras instituciones como las del sector público, no priorizaban en procesos de formación docente relacionados con la innovación tecnológica lo cual se evidencia en la deficiencia del uso de las TIC por lo que a decir de Aguilar-Gordón y Chamba (2019) se constata que hasta antes de la pandemia "los docentes son conscientes de que a pesar de vivir en una aldea global y en una era digital no logran adaptarse a las exigencias de la nueva realidad" (p. 115).

La teleeducación no causó dificultades de aprendizaje en comunidades educativas con un escenario autónomo con respecto al uso de las TIC, sin embargo, la situación fue grave para aquellas instituciones con insuficientes recursos tecnológicos y con una nula capacitación docente. En el conversatorio Adaptabilidad en la educación en tiempos de COVID llevado a cabo (en el mes de julio de 2020) por la radio online de la Universidad Politécnica Salesiana, se manifestó que "el sistema online está funcionando más en el sector particular, el sistema fiscal tiene otra forma de funcionar, (...) a través de fichas de autoaprendizaje... que llegan a los estudiantes una vez a la semana" (p. 1). Tales hechos dejan entrever la desigualdad social que evidencia que el aprendizaje no es adquirido de la misma manera, y posiblemente, en el futuro próximo, es probable que se eleven los índices de analfabetismo, el número de deserción escolar y la consecuente disminución en la cobertura educativa. 
En el caso del sistema educativo ecuatoriano, el Diario El Universo (2020) expone que "existen 3 millones de estudiantes de colegios y escuelas fiscales inscritos en 150 mil centros de enseñanza, de ellos, 2 millones (...) tienen posibilidad de conectividad; pero no corren con esa misma suerte 1 millón de estudiantes" (p. 3), quienes no cuentan con dispositivos digitales adecuados para el proceso de enseñanza - aprendizaje; incluso, aunque la mayoría de las familias poseen un dispositivo celular no todos ellos se encuentran actualizados.

Según el Diario El Universo (2020) la Corporación Nacional de Telecomunicaciones (CNT) busca obtener "el acceso a internet fijo para 943 mil estudiantes; otra, que a través de telefonía móvil llegar a 776 estudiantes en 605 parroquias; activar los 800 infocentros para el acceso a través de plantas wifi” (p. 5), o transmitir programas educativos acordes a los niveles de educación en canales del gobierno, sobre todo en aquellos lugares donde la cobertura del internet es casi nula, no se dispone de dispositivos digitales adecuados para el proceso de aprendizaje o no han utilizado teléfonos inteligentes o computadoras.

La formación educativa ocurre de manera fragmentada pues existen escenarios donde más de tres personas necesitan ingresar a sus entornos de aprendizaje, sin embargo, la falta de recursos obliga los padres de familia a colocar turnos de ingreso a la plataforma virtual, negando al otro la posibilidad de continuar con normalidad sus estudios, tales condiciones traen consigo afectaciones psicológicas a los padres de familia, a los educandos y a los docentes. En el caso de los padres de familia, su preocupación se enfoca en la falta de dinero para adquirir dispositivos que propicien el aprendizaje; en el caso de los educandos, su preocupación se centra en pasar el año; por esta razón, en este periodo de pandemia, los niveles de suicidio de niños y adolescentes es más alta en comparación con otras épocas del año. En el caso de los docentes, los niveles de estrés radican en el hecho de buscar soluciones para que cada estudiante adquiera los conocimientos básicos, desarrolle habilidades y destrezas cognitivas, procedimentales y actitudinales que permitan alcanzar una formación integral con aprendizajes significativos y útiles para toda la vida.

A esto se suma, la falta de preparación de los padres de familia en la formación escolarizada de sus hijos de los niveles iniciales y de educación básica, quienes demandan mayor tiempo y dedicación por parte del docente y de los padres de familia. El proceso de enseñanza en estos niveles presenta ciertos grados de complejidad para los docentes. Los educandos debido a su edad se distraen con facilidad, razón por la cual se requiere la presencia de los padres, en palabras de Villanueva (2020) "en el caso de los niños de inicial y primeros grados si no estaban con un adulto era difícil seguir la clase de forma fluida (TV, web, radio, etc.)" (p. 6), el problema parte de la impaciencia de la mayoría de los padres quienes al no estar acostumbrados a acompañar el proceso de aprendizaje de sus hijos acumulan exceso de estrés, desarrollan altos índices de violencia y de agresión.

La poca formación académica de los padres y la incomprensión de las tareas son causantes de impaciencia y de estrés que dan como resultado aprendizajes incompletos en el educando. Esto se complica más cuando se ha constatado que la mayor parte de padres de familia tienen que cumplir con sus responsabilidades laborales, supervisar las tareas educativas de sus hijos y a la vez estar pendientes de las actividades del hogar.

Además de lo antes referido, una de las problemáticas sociales por las cuales atravesaron varias familias a nivel mundial, fueron los despidos masivos, el factor económico se convirtió en prioridad por ser una necesidad básica para la sobrevivencia; esto se impuso al proceso educativo de varios niños y jóvenes. En el caso ecuatoriano, debido al impacto 
socioeconómico, aumentaron los niveles de deserción escolar. La situación económica impide el apoyo de los padres y lanza a algunos estudiantes hacia las calles para buscar un trabajo estable que permita cubrir las necesidades básicas del hogar. En el país, hay varias familias con trabajos informales que paulatinamente dejaron de ser rentables durante la pandemia, aspecto que aumentó los índices de pobreza, de mendicidad y de delincuencia. Conforme manifiesta Ola (2020), "muchos jóvenes están yendo a trabajar al campo, como se detuvo la actividad comercial ahora tienen que ver qué hacer, interesa más el tema de proveer a la familia que seguir en los estudios" (p. 1). Asimismo, la falta de recursos tecnológicos, la poca formación en uso de las TIC por parte de padres y educandos obstaculiza la formación académica.

Para Ola (2020) uno de los factores de deserción escolar se debe al temor al contagio, de modo que, regresar a la normalidad tendrá varias repercusiones por lo que algunas familias prefieren que sus hijos pierdan el año antes de enviarlos nuevamente a los establecimientos educativos. La infraestructura de los establecimientos educativos no reúne las condiciones necesarias para hacer realidad el distanciamiento social e inclusive, el hecho de que los padres hayan permanecido junto a sus hijos e hijas durante el aislamiento puede ser un factor viable que impida el retorno a los ambientes escolares presenciales. De acuerdo con Ola (2020) todo esto constituye "temores relacionados con su capacidad de retomar el proceso de aprendizaje, especialmente para quienes no han tenido acceso a programas de educación a distancia durante el período de confinamiento y se han desvinculado de sus estudios y la escuela" (p. 1).

Las luchas contra la desigualdad social, la discriminación, la violencia cultural, el rechazo constante antes de la pandemia parecen agudizarse durante este periodo. Si bien es cierto, el proceso del sistema educativo siguiendo una línea tradicional intentaba generar un espacio simétrico y democratizador; con la pandemia, el sistema educativo se convierte en foco de inequidades, pues a él solo accede quien tiene las posibilidades económicas y digitales, quienes no necesitan de atención a necesidades educativas especiales. El tiempo y la cantidad de educandos detrás de una pantalla dificultan al maestro realizar adaptaciones curriculares adecuadas. La formación educativa en espacios virtuales se ha convertido en un lujo al que pocos pueden acceder y en poco se verá que el capital cultural estará concentrado en manos de una élite intelectual que llegará a formar parte del sector dominante.

\section{RETOS DEL APRENDIZAJE VIRTUAL EN TIEMPOS DE PANDEMIA}

La telemática como medio de producción en las sociedades actuales afectó los horarios de trabajo, de educación y de convivencia. Los niveles de ansiedad y estrés generados por la pandemia y el confinamiento plantean una serie de retos al sistema educativo, mismo que deberá reinventarse y replantearse sobre el tipo de aprendizaje que se está creando a partir del uso de los entornos virtuales.

El proceso de aprendizaje en tiempos de pandemia es un proceso de ensayo-error, sus resultados serán evidentes en el futuro. El tipo de aprendizaje generando por los espacios virtuales es aún precario si se considera que éste inicio en la última etapa del año lectivo, pero a pesar de ello, se pudo notar que los contenidos no pudieron ser debidamente profundizados, no se hizo seguimiento del desarrollo de competencias de los estudiantes, 
el uso de las plataformas virtuales y/o las aplicaciones digitales no contribuyeron a la consolidación de los aprendizajes debido a la falta de conocimiento del funcionamiento de las mismas, las evaluaciones abiertas, las actividades grupales no pudieron ser fueron abiertas, es decir, en la mayoría de los casos, la evaluación se redujo a un trabajo grupal e irreflexivo que no evidenció el desarrollo de competencias. Algunos educandos no poseían recursos ni espacios adecuados para el estudio, no se buscaron soluciones para quienes no tenían acceso a internet y/o a dispositivos digitales, el exceso de flexibilidad para la presentación de tareas también perjudicó la calidad de los aprendizajes.

A pesar de antes afirmado, según Ola (2020) "la pandemia ha dejado al descubierto que el uso de la tecnología es vital en el campo de la educación” (p. 1). De allí que, el retomar el proceso de enseñanza-aprendizaje en modalidad virtual procura producir un aprendizaje autónomo y significativo mediante el uso de herramientas digitales interactivas gratuitas como classroom, zoom, pizarras virtuales, kahoot, to.mi digital, genially, entre otros.

Sin embargo, es importante comprender el sentido y el significado del uso de la tecnología en la educación sin perder de vista que éste depende de la perspectiva del sujeto que la utiliza y la valora, misma que a decir de Aguilar (2011) puede ser vista desde las siguientes tres aristas de reflexión: "1. Desde la apreciación subjetiva de un hecho tecnológico; 2. Desde la descripción objetiva de un proceso tecnológico; 3. Desde los resultados, productos, metas y objetivos alcanzados" (p.130). Aspectos que obligan a los involucrados a realizar un proceso de concientización previo, durante y posterior a la aplicación de cada uno de los insumos tecnológicos en el aula con miras a lograr la transformación del individuo y de la sociedad en general.

El reto de los docentes no se reduce únicamente a la transmisión de contenidos sino que va más allá, debe trabajar emociones con niños y adolescentes, siendo una forma atípica de aprendizaje (Villanueva, 2020). Los contenidos a transmitir deben partir desde la transdisciplinariedad, relacionando temas educativos con la realidad que aqueja a toda la humanidad, estableciendo comparaciones con la situación de otros países, manejando estadísticas en tiempo real, creando foros de discusión, contrastando opiniones con la finalidad de desarrollar las diferentes competencias en el sujeto que aprende. Es preciso hacer una educación realmente situada en la que se consideren íntegramente a los sujetos y sus contextos. El reto de los docentes es transformar el espacio virtual asimétrico en un espacio simétrico donde cada una de las partes (educando-educador) intervenga en igualdad de condiciones en los foros, discusiones o participaciones. De esta forma es factible generar empatía, confianza, autonomía y capacidad de emprendimiento como elementos necesarios para consensuar sentidos y significados de vida en relación con el mundo y con los otros iguales.

El manejo del espacio virtual en el proceso de enseñanza y aprendizaje en tiempos de pandemia exige transformaciones en la forma de ser, de pensar y de actuar de los sujetos involucrados, conlleva nuevas formas de enseñar y de aprender. Así, en este contexto, conforme a Capdet (2011) el docente es "un hábil mediador de conflictos que con su acción contribuye decisivamente a negociar un significado consensuado y a mantener la estabilidad y el equilibrio del contexto en el que están inmersos" (p. 57), mediante la creatividad.

Otro reto importante del aprendizaje virtual en tiempos de la emergencia sanitaria es para la familia, los adultos y/o representantes de niños y jóvenes quienes se han convertido en el centro fundamental del proceso educativo, ellos son los responsables directos del control y del manejo de actitudes, comportamientos y aprendizajes en la nueva realidad 
que ha correspondido vivir a la sociedad de los últimos tiempos, a ellos corresponde la identificación de estilos de aprendizaje, el control de tiempo-espacio, el seguimiento de las habilidades relacionales, socioemocionales, el acompañamiento y el cultivo de valores con las directrices que proporciona la institución educativa. Uno de los desafíos para el cumplimiento de la función de la familia en el proceso de aprendizaje es la comunicación permanente para potenciar la participación, la colaboración, la solución de problemas, el uso equilibrado de redes sociales y demás insumos tecnológicos, la emisión de ideas, sentimientos y pensamientos conforme a necesidades e intereses de los niños y jóvenes. Es tarea de la familia estimular y garantizar el bienestar físico, emocional y psicológico de los niños y adolescentes.

\section{CONCLUSIÓN}

La emergencia sanitaria aceleró el paso en el reemplazo de escenarios de aprendizaje presencial a escenarios de aprendizaje virtual, tal proceso venía gestándose de manera lenta desde el inicio del siglo XXI, en ese periodo se dio lugar a la educación no formal e informal mediante la inserción de las nuevas tecnologías de comunicación e información, herramientas que conceden al educando horarios flexibles, contenidos asequibles, comodidad de estudio entre otras cosas. Con la pandemia, el rol de cada integrante de la comunidad educativa sufre ciertas alteraciones, siendo el aprendiz el protagonista principal en el proceso educativo.

No obstante, el cambio abrupto a una realidad virtual deja entrever las precariedades en el diseño de políticas para la era digital; debido a la crisis económica (propia de la época) la gran mayoría de la población experimenta dificultades para acceder a este tipo de escenarios de aprendizaje por la falta de dispositivos digitales, falta de conectividad, escaso conocimiento sobre el uso de las TIC, un limitado capital cultural en los padres, etc. Además de la emergencia sanitaria, la situación se complica más con el despido masivo de trabajadores formales y las limitaciones para trabajadores informales, aspectos que de una $\mathrm{u}$ otra manera inciden en los procesos educativos. Las desigualdades son evidentes entre aquellos que tienen posibilidades económicas y aquellos que a diario buscan medios para vivir. El factor económico y la brecha digital se han convertido en los causantes de la deserción escolar de los últimos tiempos.

El proceso de aprendizaje en tiempos de pandemia es un reto para toda la comunidad educativa, el cambio repentino de escenarios presenciales de aprendizaje a un escenario virtual limita el contacto social, tal limitación puede traer consigo varias consecuencias, por ejemplo, la relación directa entre sujetos y dispositivos digitales evitan reconocer las emociones y sentimientos de los otros. Asimismo, las realidades educativas de los educandos difieren con cada sujeto, existiendo educandos con necesidades educativas especiales en los que el proceso de aprendizaje es incompleto debido a la inadecuada adaptación curricular por parte de los docentes, quienes a su vez tienen dificultades para adaptarse a la realidad virtual.

Cabe resaltar que, el uso correcto de los recursos tecnológicos en el proceso de enseñanza- aprendizaje pueden generar autonomía, criticidad y un aprendizaje participativo en el ser humano que aprende. El paso del aprendizaje en escenarios presenciales al aprendizaje virtual en tiempos de pandemia constituye un reto para la comunidad educativa, 
el rol del docente, los contenidos, el desarrollo de competencias y la evaluación deben partir desde la realidad del confinamiento social, para ello es preciso prestar atención al trabajo de las emociones para no caer en individualismos o en inútiles cosificaciones.

\section{REFERENCIAS BIBLIOGRÁFICAS}

Aguilar, F. (2011). Reflexiones filosóficas sobre la tecnología y sus nuevos escenarios. En Sophia. Colección de Filosofía de la Educación, (11), 123-174. Doi: https://doi.org/10.17163/soph. n11.2011.06

Aguilar-Gordón, F. \& Chamba, A. P. (2019). Reflexiones sobre la Filosofía de la Tecnología en los procesos educativos. CONRADO. Cienfuegos, XV(70), 109-119.

http://conrado.ucf.edu.cu/index.php/conrado

Capdet, D. (2011). Conectivismo y Aprendizaje informal: Análisis desde el punto de vista de una sociedad en proceso de transformación.

El Universo. (24 de Abril de 2020). Un millón de estudiantes sin acceso a educación virtual durante la emergencia sanitaria. El Universo

https://www.eluniverso.com/noticias/2020/04/24/nota/7822794/millon-estudiantes-accesoeducacion-virtual-durante-emergencia

Martí Castro, I. (2003). Aprendizaje-Virtual. En Diccionario Enciclopédico de Educación. Grupo Editorial Ceac S. A. (LEXUS).

Narvarte, M. (2008). Soluciones Pedagógicas en el Aula. Landeira Edicaciones S. A.

Ola, A. L. (30 de junio de 2020). Coronavirus: La deserción escolar es una amenaza pospandemia. https://forbescentroamerica.com/2020/06/30/coronavirus-la-desercion-escolar-es-unaamenaza-pospandemia/

Papalia, D., Feldman, R. \& Martorell, G. (2012). Desarrollo Humano (Duodécima ed.). McGrawHill.

Tennuto, M., Klinoff, A. \& Boan, S. (2003). Escuela para Maestros. Enciclopedia de Pedagogía Práctica. Círculo Latino Austral. Lexus .

UPS, U. P. (15 de Julio de 2020). Adaptabilidad en la educación en tiempos de COVID. https://www. ups.edu.ec/noticias?articleId=17026044

Villanueva, L. (Consultado el 15 de Septiembre de 2020). El gran reto de la educación virtual en tiempos de pandemia. Fundación Wiese. Blog: https://www.fundacionwiese.org/blog/es/elgran-reto-de-la-educacion-virtual-en-tiempos-de-pandemia/

Zizek, S. et al. (2008). Arte, ideología y capitalismo. Círculo de Bellas Artes. 
